University of New Hampshire

University of New Hampshire Scholars' Repository

\title{
$5-2015$
}

\section{Energetic, relativistic and ultra-relativistic electrons: Comparison of long-term VERB code simulations with Van Allen Probes measurements}

\author{
A. Drozdov \\ University of California - Los Angeles \\ Y. Y. Shprits \\ University of California - Los Angeles \\ K. Orlova \\ University of California - Los Angeles \\ A. C. Kellerman \\ University of California - Los Angeles \\ D. A. Subbotin \\ University of California - Los Angeles
}

See next page for additional authors

Follow this and additional works at: https://scholars.unh.edu/physics_facpub

Part of the Astrophysics and Astronomy Commons

\section{Recommended Citation}

Drozdov, A. Y., Y. Y. Shprits, K. G. Orlova, A. C. Kellerman, D. A. Subbotin, D. N. Baker, H. E. Spence, and G. D. Reeves (2015), Energetic, relativistic, and ultrarelativistic electrons: Comparison of long-term VERB code simulations with Van Allen Probes measurements, J. Geophys. Res. Space Physics, 120, 3574-3587, doi:10.1002/ 2014JA020637

This Article is brought to you for free and open access by the Physics at University of New Hampshire Scholars' Repository. It has been accepted for inclusion in Physics Scholarship by an authorized administrator of University of New Hampshire Scholars' Repository. For more information, please contact Scholarly.Communication@unh.edu. 


\section{Authors}

A. Drozdov, Y. Y. Shprits, K. Orlova, A. C. Kellerman, D. A. Subbotin, D. N. Baker, Harlan E. Spence, and Geoffrey Reeves 


\section{Journal of Geophysical Research: Space Physics}

\section{RESEARCH ARTICLE \\ 10.1002/2014JA020637 \\ Special Section: \\ Energetic, relativistic, and ultrarelativistic electrons: Comparison of long-term VERB code simulations with Van Allen Probes measurements}

New perspectives on Earth's radiation belt regions from the prime mission of the Van Allen Probes

Key Points:

- VERB code reproduces the dynamics of relativistic multi-MeV electrons

-Whistler waves and radial diffusion cannot explain their observed losses

- Scattering by EMIC waves is the most likely explanation for the observed losses

Correspondence to:

A. Y. Drozdov,

adrozdov@ucla.edu

\section{Citation:}

Drozdov, A. Y., Y. Y. Shprits, K. G. Orlova, A. C. Kellerman, D. A. Subbotin, D. N. Baker, H. E. Spence, and G. D. Reeves (2015),

Energetic, relativistic, and ultrarelativistic electrons: Comparison of long-term VERB code simulations with Van Allen Probes measurements, J. Geophys. Res. Space Physics, 120, 3574-3587, doi:10.1002/ 2014JA020637.

Received 24 SEP 2014 Accepted 13 APR 2015 Accepted article online 16 APR 2015 Published online 16 MAY 2015

C2015. American Geophysical Union. Al Rights Reserved.

\author{
A. Y. Drozdov ${ }^{1}$, Y. Y. Shprits ${ }^{1,2}$, K. G. Orlova ${ }^{1}$, A. C. Kellerman ${ }^{1}$, D. A. Subbotin ${ }^{1}$, D. N. Baker ${ }^{3}$, \\ H. E. Spence ${ }^{4}$, and G. D. Reeves ${ }^{5}$
}

${ }^{1}$ Department of Earth, Planetary, and Space Sciences, University of California, Los Angeles, California, USA, ${ }^{2}$ Massachusetts Institute of Technology, Cambridge, Massachusetts, USA, ${ }^{3}$ Laboratory for Atmospheric and Space Physics, University of Colorado, Boulder, Colorado, USA, ${ }^{4}$ Institute for the Study of Earth Oceans and Space, University of New Hampshire, Durham, New Hampshire, USA, ${ }^{5}$ Space Science and Applications Group, Los Alamos National Laboratory, Los Alamos, New Mexico, USA

Abstract In this study, we compare long-term simulations performed by the Versatile Electron Radiation Belt (VERB) code with observations from the Magnetic Electron lon Spectrometer and Relativistic Electron-Proton Telescope instruments on the Van Allen Probes satellites. The model takes into account radial, energy, pitch angle and mixed diffusion, losses into the atmosphere, and magnetopause shadowing. We consider the energetic $(>100 \mathrm{keV})$, relativistic $(\sim 0.5-1 \mathrm{MeV})$, and ultrarelativistic $(>2 \mathrm{MeV})$ electrons. One year of relativistic electron measurements ( $\mu=700 \mathrm{MeV} / \mathrm{G}$ ) from 1 October 2012 to 1 October 2013 are well reproduced by the simulation during varying levels of geomagnetic activity. However, for ultrarelativistic energies $(\mu=3500 \mathrm{MeV} / \mathrm{G})$, the VERB code simulation overestimates electron fluxes and phase space density. These results indicate that an additional loss mechanism is operational and efficient for these high energies. The most likely mechanism for explaining the observed loss at ultrarelativistic energies is scattering by the electromagnetic ion cyclotron waves.

\section{Introduction}

Trapped relativistic electrons in the radiation belts exhibit a two-zone structure separated by a gap, referred to as the slot region [Van Allen and Frank, 1959; Vernov et al., 1969; Russell and Thorne, 1970]. The inner belt (below 2.0 Earth radii) is stable, while the outer belt (above 3.0 Earth radii) can be very dynamic [Rothwell and Mcllwain, 1960] and is strongly driven by geomagnetic activity. The dynamic evolution of the radiation belts is a result of competition between various acceleration and loss processes [Reeves et al., 2003; Shprits et al., 2008a, 2008b; Millan and Baker, 2012; Turner et al., 2014].

Consideration of phase space density (PSD) in terms of adiabatic invariant coordinates enables the differentiation between adiabatic and nonadiabatic processes [Green and Kivelson, 2001; Shprits et al., 2008a]. Quasi-linear theory is a common approach for modeling the electron PSD dynamics [Kennel and Engelmann, 1966; Schulz and Lanzerotti, 1974]. The diffusion equation describing the variation of PSD in time can be derived assuming that the collisionless charged particles in the ambient magnetic field undergo resonant interactions with relatively small-amplitude incoherent electromagnetic waves. The radiation belt dynamics have been modeled as simple radial diffusion with loss [Brautigam and Albert, 2000; Shprits et al., 2005; Tu et al., 2009]. However, local processes are also important and in some cases can be modeled using pitch angle diffusion only, for example, in the case of studying scattering of the electrons by hiss waves inside the plasmasphere [Shprits et al., 2006b; Thorne et al., 2013a], or a combination of energy and pitch angle diffusions using various types of waves [Shprits et al., 2006a; Thorne et al., 2013b]. The comprehensive picture of the competition between acceleration and loss processes caused by radial, energy, and pitch angle diffusion can be studied using a numerical solution of the three-dimensional Fokker-Planck equation [Albert et al., 2009; Subbotin and Shprits, 2009; Su et al., 2011; Reeves et al., 2012; Tu et al., 2013; Glauert et al., 2014]. In this study, we model the long-term variation of PSD using the 3-D Versatile Electron Radiation Belt (VERB) code [Shprits et al., 2008a, 2009a; Subbotin and Shprits, 2009; Kim et al., 2011a; Kim et al., 2012; Subbotin et al., 2011a, 2011b], which incorporates a recently updated diffusion model for hiss, based on parameterizations of Orlova et al. [2014]. 
If the model has an unrealistic balance between loss and source processes, long-term simulations will either result in the disappearance of the belts or the formation of unrealistically high electron fluxes. Long-term simulations also allow us to test whether this balance between losses and source processes results in realistic dynamics of the electrons, a test that cannot be done using a single storm but requires long-term simulations. An even more challenging task, which is addressed in this study, is to reproduce the difference in behavior of particles at different energies. The physics-based VERB code allows for the inclusion of different combinations of diffusion coefficients into the model. Long-term simulations can then be performed to simulate the relative contribution of the various source and loss processes at different energies.

Recent reports of long-term simulations using VERB (or similar codes) [Subbotin et al., 2011a; Kim et al., 2012; Kim and Shprits, 2013] have shown reasonably good agreement with Combined Release and Radiation Effects Satellite (CRRES) observations at approximately MeV energies. Shprits et al. [2013] considered the September-October period in 2012, during which the three-zone structure was first observed by Van Allen Probes [Baker et al., 2013a], and showed that at ultrarelativistic energies, energization and loss processes may be very different from MeV energies. They showed that electromagnetic ion cyclotron (EMIC) waves may play an important role in scattering ultrarelativistic electrons, while acceleration by chorus and scattering by hiss may occur significantly slower at these energies. Most recently, Thorne et al. [2013b] and Reeves et al. [2013] have shown that during some storms, when convective activity is continually enhanced for several days, chorus waves may result in a significant acceleration of not only relativistic but also ultrarelativistic electrons, which is consistent with the observed peaks in phase-space density.

In this study, a long-term simulation with the VERB code (1 year starting near the beginning of the Van Allen Probes mission) is compared with in situ measurements from the Van Allen Probes Magnetic Electron lon Spectrometer (MagEIS) and Relativistic Electron-Proton Telescope (REPT) instruments. We show that consideration of the ultrarelativistic electrons requires additional understanding of the physical processes that drive the dynamics of the radiation belts.

\section{Satellite Observations}

\subsection{CRRES}

The Combined Release and Radiation Effects Satellite was launched on 20 July 1990 and was in operation until 11 October 1991. The energies, measured by The Medium Electron A (MEA) instrument [Vampola et al., 1992] installed on CRRES, are logarithmically distributed from 0.15 to $1.58 \mathrm{MeV}$, with 17 energy channels in total. We used the $1 \mathrm{MeV}$ electron measurements to explore the dynamics of the relativistic electrons and to validate our simulation with the latest version of the code that includes losses to the magnetopause and a new parameterization of hiss waves.

\subsection{Van Allen Probes}

The Van Allen Probes [Mauk et al., 2013; Stratton et al., 2014] were launched in 2012 to study the dynamical evolution of the radiation belts. The two Van Allen Probes spacecraft are equipped with an identical array of instruments suited for the monitoring of radiation belt particles and wave environment. The unprecedented energy and pitch angle resolution of the instruments provide a more comprehensive picture of the radiation belts than previously available from other spacecraft missions. Particles with energies ranging from hot to ultrarelativistic are measured using the Radiation Belt Storm Probes-Energetic particle, Composition, and Thermal plasma (RBSP-ECT) suite [Spence et al., 2013]. In the current work, we used data from the Magnetic Electron Ion Spectrometer (MagEIS) [Blake et al., 2013], which provides measurements of electrons in the energy range from $\sim 30 \mathrm{keV}$ to about $4 \mathrm{MeV}$, and Relativistic Electron-Proton Telescope (REPT) instrument [Baker et al., 2013b], which covers a range of energies from $2 \mathrm{MeV}$ to tens of MeV.

Based on pitch angle-resolved flux measurements, PSDs were calculated for a wide range of $L$ shells from $\sim 1$ to 5.5. The $L$ shell parameter [Roederer, 1970] and $K$ invariant (geometric interpretation of the second invariant [Schulz and Lanzerotti, 1974]) were calculated based on the Tsyganenko 89 (T89) field model [Tsyganenko, 1989]. The pitch angle distribution was interpolated on a uniform grid with a step of $5^{\circ}$. The data from both satellites RBSP-A and RBSB-B were used. In the points where observations from both satellites are available, the measurements were averaged. 
During the period of operation of the Van Allen Probes mission, the energy channels and gain definitions for the MagEIS instrument were adjusted (S. Claudepierre, personal communication, 2014). Estimation of the fluxes at fixed energy was determined by interpolation of the gridded data.

\section{VERB Code}

The radiation belts' dynamics and evolution can be modeled as the phase-averaged diffusion of electron PSD in terms of three adiabatic invariants (I), which is described by the Fokker-Planck equation [Schulz and Lanzerotti, 1974]:

$$
\frac{\partial f}{\partial t}=\sum_{i, j=1}^{3} \frac{\partial}{\partial l_{i}} D_{l_{i} l_{j}} \frac{\partial f}{\partial l_{j}}
$$

where $f$ is the PSD, $D_{l_{i} l_{j}}$ are the diffusion coefficients, and the $i$ and $j$ are the summation indices.

The equation can be written in terms of the $L$ shell, momentum $p$, and equatorial pitch angle of electrons $\alpha$ [Shprits et al., 2008a; Subbotin and Shprits, 2009]:

$$
\begin{aligned}
\frac{\partial f}{\partial t}= & \left.\mathrm{L}^{* 2} \frac{\partial}{\partial \mathrm{L}^{*}}\right|_{\mu, J}\left(\left.D_{\mathrm{L}^{*} \mathrm{~L}^{\mathrm{L}^{*}}} \frac{\partial f}{\partial \mathrm{L}^{*}}\right|_{\mu, J}\right) \\
& +\left.\frac{1}{T(\alpha) \sin (2 \alpha)} \frac{\partial}{\partial \alpha}\right|_{p, \mathrm{~L}^{*}} T(\alpha) \sin (2 \alpha)\left(\left.D_{\alpha \alpha} \frac{\partial f}{\partial \alpha}\right|_{p, \mathrm{~L}^{*}}+\left.D_{p \alpha} \frac{\partial f}{\partial p}\right|_{\alpha, \mathrm{L}^{*}}\right) \\
& +\left.\frac{1}{p^{2}} \frac{\partial}{\partial p^{2}}\right|_{\alpha, \mathrm{L}^{*}} p^{2}\left(\left.D_{p p} \frac{\partial f}{\partial p}\right|_{\alpha, \mathrm{L}^{*}}+\left.D_{p \alpha} \frac{\partial f}{\partial \alpha}\right|_{p, \mathrm{~L}^{*}}\right)-\frac{f}{\tau_{l c}}
\end{aligned}
$$

where $\mu$ and $J$ are the first and second adiabatic invariants, respectively, and $\mathrm{L}^{*}$ is an adiabatic invariant ( $\mathrm{L}$ shell based on a dipole magnetic field model), which is inversely proportional to the third adiabatic invariant $\Phi$ and is constant along the particle's drift path. $D_{\alpha \alpha}$ and $D_{p p}$ are the bounce-averaged pitch angle and momentum diffusion coefficients, respectively; $D_{\alpha p}$ are the mixed diffusion terms; $T(\alpha)$ is a function related to the particle's bounce time; and $D_{L^{*} L^{*}}$ is the radial diffusion coefficient. The term $f / \tau_{l c}$ represents electron losses. Parameter $\tau_{l c}$ is a loss rate which is equal to a quarter bounce period inside of the loss cone and infinity outside of the loss cone. In this study, we neglect the potentially important cross L pitch angle diffusion [O'Brien, 2014].

Wave-particle interactions may violate all three adiabatic invariants and can result in the radial transport, local acceleration, and loss of electrons. The numerical simulation takes into account radial diffusion caused by ultralow frequency waves, as well as pitch angle, energy, and mixed diffusion caused by whistler waves. The Kp-dependent radial diffusion coefficient is adopted from Brautigam and Albert [2000]. The parameters for the chorus, VLF, and lightning waves are taken from Subbotin et al. [2011a]. For the hiss waves, we used realistic parameters from Orlova et al. [2014] with the frequency range of $100-2000 \mathrm{~Hz}$ and a peak power at $550 \mathrm{~Hz}$. Using data from the CRRES wave experiment, Orlova et al. [2014] developed quadratic fits to hiss amplitude as a function of $\mathrm{L}$ shell, $K p$, and latitude for the dayside and nightside. It was considered that hiss waves become more oblique as they propagate along the field line following Agapitov and Artemyev [2013] and Thorne et al. [2013a]. The frequency spectrum of hiss waves was assumed to be Gaussian with the same parameters as in previous studies [Shprits et al., 2009a; Thorne et al., 2013a]. The latitudinal-dependent model of plasmaspheric density was taken from Denton et al. [2004, 2006]. The diffusion coefficients related to lightning whistlers, hiss, and chorus waves were averaged over magnetic local time (MLT) and scaled using the $K p$ index. We assume that above the plasmapause, which is calculated following Carpenter and Anderson [1992], scattering is dominated by the chorus waves, while inside the plasmasphere, scattering is provided by hiss waves. The amplitudes of both types of whistler mode waves are parameterized by the $K p$ index.

The computational grid has $101 \times 91 \times 46$ points for energy, pitch angle, and radial diffusion, respectively. Energy and pitch angle grid points are distributed logarithmically. The initial conditions are calculated as the steady state solution for the radial diffusion equation. The solution of equation (2) also requires six 
boundary conditions: an upper and lower value of the electron PSD for each of the variables. The lower boundary for energy diffusion varies with the radial distance. At $L^{*}=5.5$, the lower boundary is set at $10 \mathrm{keV}$. Energy at which lower boundary conditions on the energy diffusion operator are set up is increasing with decrease of the radial distance according to adiabatic transport from $\mathrm{L}^{*}=5.5$ to the point of interest. The upper energy boundary $(10 \mathrm{MeV})$ can be set to zero, because the flux at this energy is very low. However, we found that the simulation maintains a very low value of PSD at the upper boundary and is more stable when we set up the derivative with respect to energy to zero, instead of setting up the value of PSD to zero at this boundary. The PSD derivatives for both pitch angle boundaries are set to zero to allow for simulation of strong and weak diffusions at $0^{\circ}$ [Shprits et al., 2009b] and flat distribution at $90^{\circ}$ [Horne et al., 2003]. PSD at the lower radial boundary $\left(\mathrm{L}^{*}=1\right)$ is set to zero and represents losses into the atmosphere.

Previous studies have set up the outer boundary conditions at $L^{*}=7$ [Shprits et al., 2009a], at $L^{*}=6$ [Tu et al., 2009], and at $L^{*}=5.5$ [Kim et al., 2011b] and showed that the simulations with the outer boundary closer to the Earth can better reproduce fluxes in the heart of the radiation belts. The improved accuracy of simulations is likely due to the fact that at $L^{*}=5.5$, the outer boundary can better account for the source of the lower energy seed population. The PSD required for the upper radial boundary $\left(\mathrm{L}^{*}=5.5\right)$ condition is computed using the long-time averaged shape of PSD spectrum measured at geosynchronous orbit following Subbotin et al. [2011a]. The average PSD as a function of energy is transferred from geosynchronous to $L^{*}=5.5$ as a steady state solution of the radial diffusion equation. The satellite observations at $L^{*}=5.5$ are used to obtain the variation of the PSD in time for the selected energy. The ratio of the averaged and measured PSD at the same energy is a scaling factor that defines the energy-independent variation of the upper radial boundary.

We use the T89 magnetic field model to calculate the dependence of the magnetopause location on $\mathrm{Kp}$ and pitch angle. The PSD outside the magnetopause location is set to zero. A more accurate treatment of loss requires an energy-dependent loss mechanism, because the magnetopause shadowing effect is also dependent on drift period and will be accounted in future studies.

\section{Comparison Between Simulation and Observations}

\subsection{CRRES Relativistic Electron Flux}

To ensure that the latest changes in the code do not entail deterioration of the simulation result, we configure the test simulation based on CRRES data observations for the same period and simulation conditions used in previous studies [Subbotin et al., 2011a; Kim et al., 2012]. The $1 \mathrm{MeV}$ flux variation for the period from 29 July 1990 to 6 November 1990 was used to establish the upper radial boundary condition. Figure 1 shows the comparison between 100 days of CRRES $1 \mathrm{MeV}$ flux observations (Figure 1a), VERB simulation (Figure 1b), and logarithmic difference (Figure 1c).

The simulation begins near the 29 July 1990 storm. Although there was not enough data to fully represent the upper boundary radial conditions for this storm period, the VERB code reproduces electron acceleration close to $L^{*}=3$. As shown by Subbotin et al. [2011a], this is the result of radial and energy diffusions. During the next two close storms at the end of August 1990, the model shows electron acceleration, which is in relatively good agreement with the measurements. On 26 August 1990, CRRES observed an electron depletion above $\mathrm{L}^{*}=4.5$, which is likely a result of the loss to the magnetopause and the outward radial diffusion. The loss to the magnetopause is influenced by the adiabatic effects that cannot be currently modeled by the VERB code [e.g., Kim et al., 2011a]. The model shows a similar depletion at $\mathrm{L}^{*}>4$.9. The difference between real magnetic field variation and the field modeled by Tsyganenko 89 (which was used to calculate $L^{*}$ for the observations) may be responsible for this minor distinction between the data and simulation. The 10 October 1990 storm begins with a dropout in electron fluxes. During the recovery phase, the fluxes were restored and the electrons accelerated, followed by an injection of electrons several days after the storm. The VERB simulation shows similar behavior. A more realistic plasmapause position [e.g., O'Brien and Moldwin, 2003], MLT-dependent plasmapause location, and improved chorus wave model may reduce the quantitative difference between the observations and the model. 


\section{AGU. Journal of Geophysical Research: Space Physics}

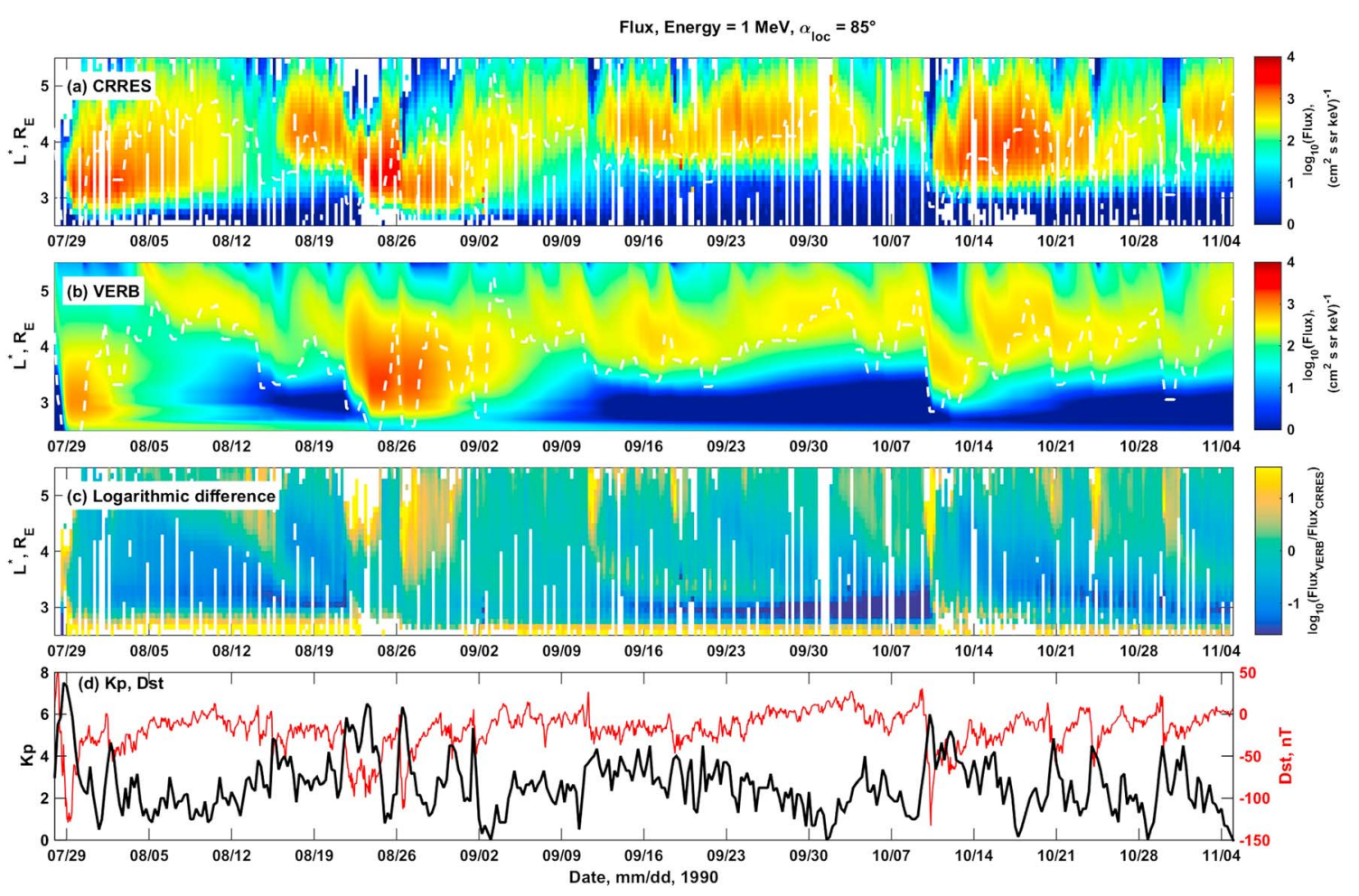

Figure 1. Electron flux at $1 \mathrm{MeV}, \alpha_{\mathrm{loc}}=85^{\circ}$ for 100 days. (a) CRRES observations, (b) VERB code simulation, and (c) logarithmic difference between simulated and observed fluxes. The white dashed line indicates the plasmapause location. (d) $K p$ (black) and Dst (red) indices variation.

In general, the VERB test simulation reproduces well the general dynamics of the radiation belts as observed by CRRES. The quantitative difference between measurement and simulation in the heart of the radiation belts mainly lies within 1 order of magnitude (Figure 1c). However, MEA observations are limited in energy and only available during 1990 and 1991.

\subsection{Relativistic Electrons From Van Allen Probes}

The RBSP-ECT suite of instruments on the Van Allen Probes covers a very large range of particle energies. Both CRRES and Van Allen Probes cover the period around the maximum phase of the solar cycle; however, the general dynamics of the cycles are very different. We performed a long-term simulation from 1 October 2012 to 1 October 2013 based on Van Allen Probes observations. The $0.9 \mathrm{MeV}$ flux channel of the MagEIS instrument and 3.6 MeV flux channel of the REPT instrument were used to provide the dynamics of the outer boundary following the method used by Brautigam and Albert [2000] for two independent simulations.

Figure 2 shows the comparison between the MagEIS observations and 1 year of modeling using the boundary conditions inferred from MagEIS observations at $\mathrm{L}^{*}=5.5$. Relativistic electron flux is shown at fixed energy of $0.9 \mathrm{MeV}$ and for local pitch angle of $85^{\circ}$ (Figures $2 \mathrm{a}$ and $2 \mathrm{~b}$ ). PSD is shown at fixed first and second invariants $\mu=700 \mathrm{MeV} / \mathrm{G}, K=0.11 R_{E}^{0.5} \mathrm{G}$ (Figures $2 \mathrm{c}$ and $2 \mathrm{~d}$ ), together with the variation of the $K p$ and Dst indices (Figure 2e).

Here we discuss the comparison of the observed and modeled dynamics of the radiation belts, chronologically. A double peak storm occurred on 9 and 13 October 2012. The simulation reproduces the electron flux acceleration and PSD radial diffusion transport during this period. During the 1 and 14 November 2012 storms, the observed and modeled fluxes and PSDs show the depletion in the outer electron belt. The long period of relatively quiet geomagnetic activity up to 1 March 2013 contains several injections and decays that are qualitatively reproduced by the VERB code. The observed trend of sudden flux enhancement on 1 March 2013 followed by the 17 March 2013 storm is also seen in the VERB code simulation. The model shows the dropout during the main phase of the 17 March 2013 storm and acceleration with additional injections during the recovery phase that is seen in the observed PSD and 

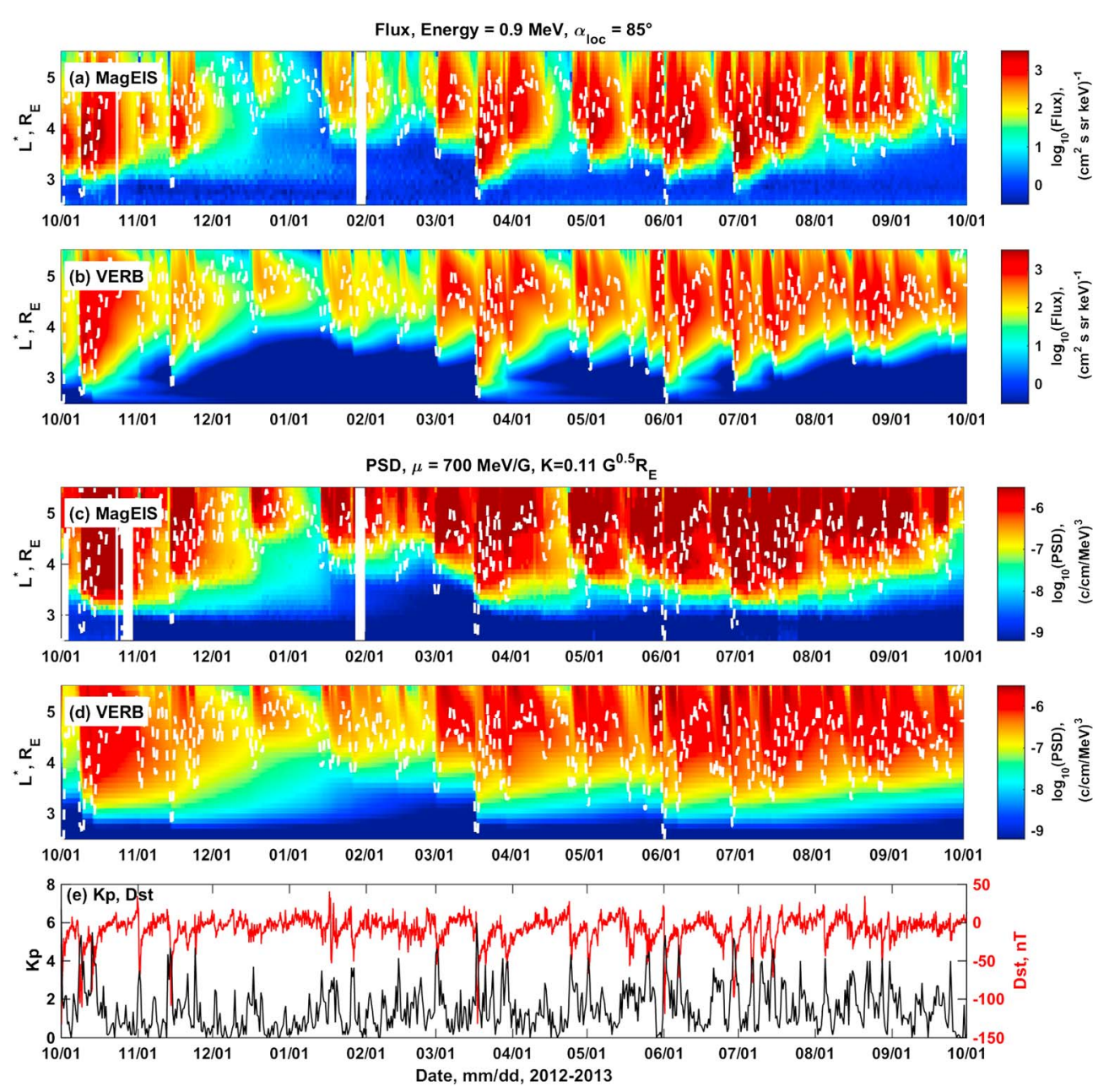

Figure 2. Relativistic electron flux at $0.9 \mathrm{MeV}, \alpha_{\mathrm{loc}}=85^{\circ}$ (a) from MagEIS observations and (b) from the simulation based on boundary conditions from MagEIS data. PSD at $\mu=700 \mathrm{MeV} / \mathrm{G}, K=0.11 R_{E}{ }^{0.5} \mathrm{G}$ from (c) MagEIS observations and (d) corresponding VERB code simulation. The white dashed line indicates the plasmapause location. (e) $K p$ (black) and $D s t$ (red) indices variation.

flux. Over approximately the next 6 months, the geomagnetic activity was mostly moderate, including several minor storms, and the outer radiation belt filled with relativistic electrons. The simulation drives several depletions and enhancements of electrons. This behavior is also seen in the observations. The storms observed on 1 and 28 June are similar to the 17 March 2013 event, beginning with a rapid dropout followed by acceleration in the recovery phase, and are reproduced by the VERB code.

The long-term simulation of global relativistic electron dynamics shows that the processes included in the VERB code provide a reasonable agreement between the simulation and the satellite measurements. The possible reasons for the quantitative difference between the data and model could be the uncertainty of the plasmapause location and plasma density model as well as chorus wave parameters, as discussed earlier. It should be noted that the particular events require detailed analysis since the global modeling is based on statistical studies of the plasma and wave parameters. Parameters such as plasma density or population of seed electrons may play a significant role for the dynamics of relativistic electrons [Thorne et al., 2013b; Tu et al., 2014; Boyd et al., 2014].

\subsection{Ultrarelativistic Electrons Observed on Van Allen Probes}

Figure 3 shows the ultrarelativistic electron flux and PSD profiles from the simulation with boundary conditions from REPT data at $\mathrm{L}^{*}=5.5$ for the same time period as in Figure 2 and comparison with satellite observations from the REPT instrument. We use fluxes at an electron energy of $3.6 \mathrm{MeV}$, a local pitch angle of $85^{\circ}$, and PSD 

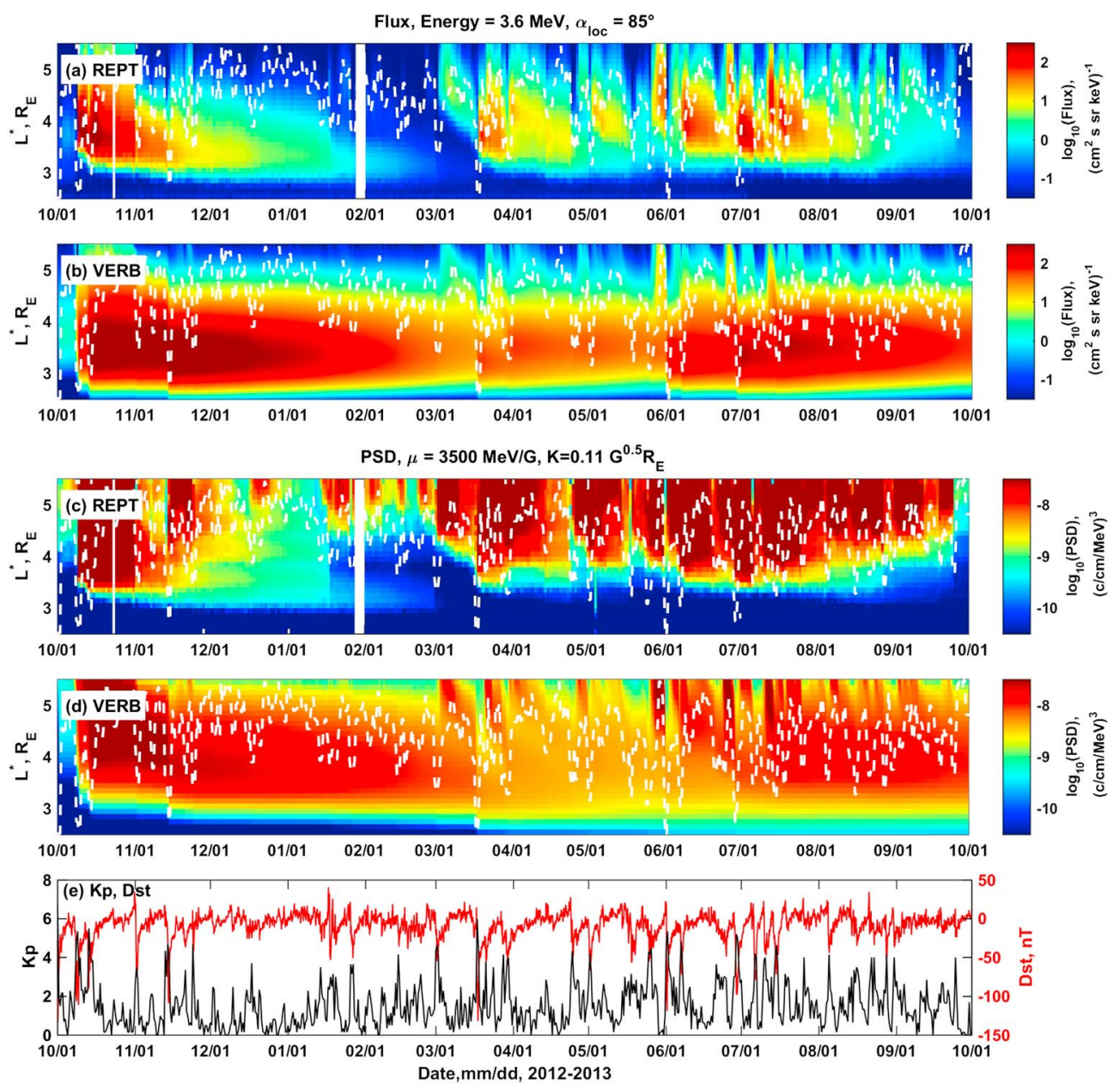

Figure 3. (a-e) Same figure as in Figure 2 but for ultrarelativistic electron flux at $3.6 \mathrm{MeV}, \alpha_{\mathrm{loc}}=85^{\circ}$ and PSD at $\mu=3500 \mathrm{MeV} / \mathrm{G}, K=0.11 R_{E}{ }^{0.5} \mathrm{G}$. The simulation based on boundary conditions from REPT data. The observed measurements are taken from the REPT instrument.

profiles at fixed first and second invariants $\left(\mu=3500 \mathrm{MeV} / \mathrm{G}, K=0.11 R_{E}{ }^{0.5} \mathrm{G}\right.$ ). The calculated flux (Figure $3 \mathrm{~b}$ ) and PSD (Figure 3d) overestimate the measurements (Figures 3a and $3 \mathrm{c}$ ) below the plasmapause. The difference is systematic in nature, as it can be clearly seen from Figure 3 that the modeled dynamics are very different from the observed dynamics of the outer belt. The simulation produces an extension in the $L$ shell's wide remnant belt, while observations only show narrow remnant belts at low $L$ shells after the 13 October 2012 storm and electron enhancement separated by clear dropouts after the 17 March 2013 storm.

\section{Discussion of Possible Loss Mechanisms}

A recent study by Thorne et al. [2013a] performed simple 1-D pitch angle scattering simulations and concluded that scattering by hiss waves was sufficient to explain the decays of ultrarelativistic electrons in the radiation belts inside the plasmasphere. To better understand if hiss waves can produce a sufficient decay of fluxes, we performed simulations based upon the truncated forms of equation (2). In order to exclude the effects of radial diffusion, we numerically solved equation (3):

$$
\begin{aligned}
\frac{\partial f}{\partial t}= & \left.\frac{1}{T(\alpha) \sin (2 \alpha)} \frac{\partial}{\partial \alpha}\right|_{p, L^{*}} T(\alpha) \sin (2 \alpha)\left(\left.D_{\alpha \alpha} \frac{\partial f}{\partial \alpha}\right|_{p, L^{*}}+\left.D_{p \alpha} \frac{\partial f}{\partial p}\right|_{\alpha, L^{*}}\right) \\
& +\left.\frac{1}{p^{2}} \frac{\partial}{\partial p^{2}}\right|_{\alpha, L^{*}} p^{2}\left(\left.D_{p p} \frac{\partial f}{\partial p}\right|_{\alpha, L^{*}}+\left.D_{p \alpha} \frac{\partial f}{\partial \alpha}\right|_{p, L^{*}}\right)-\frac{f}{\tau_{l c}}
\end{aligned}
$$



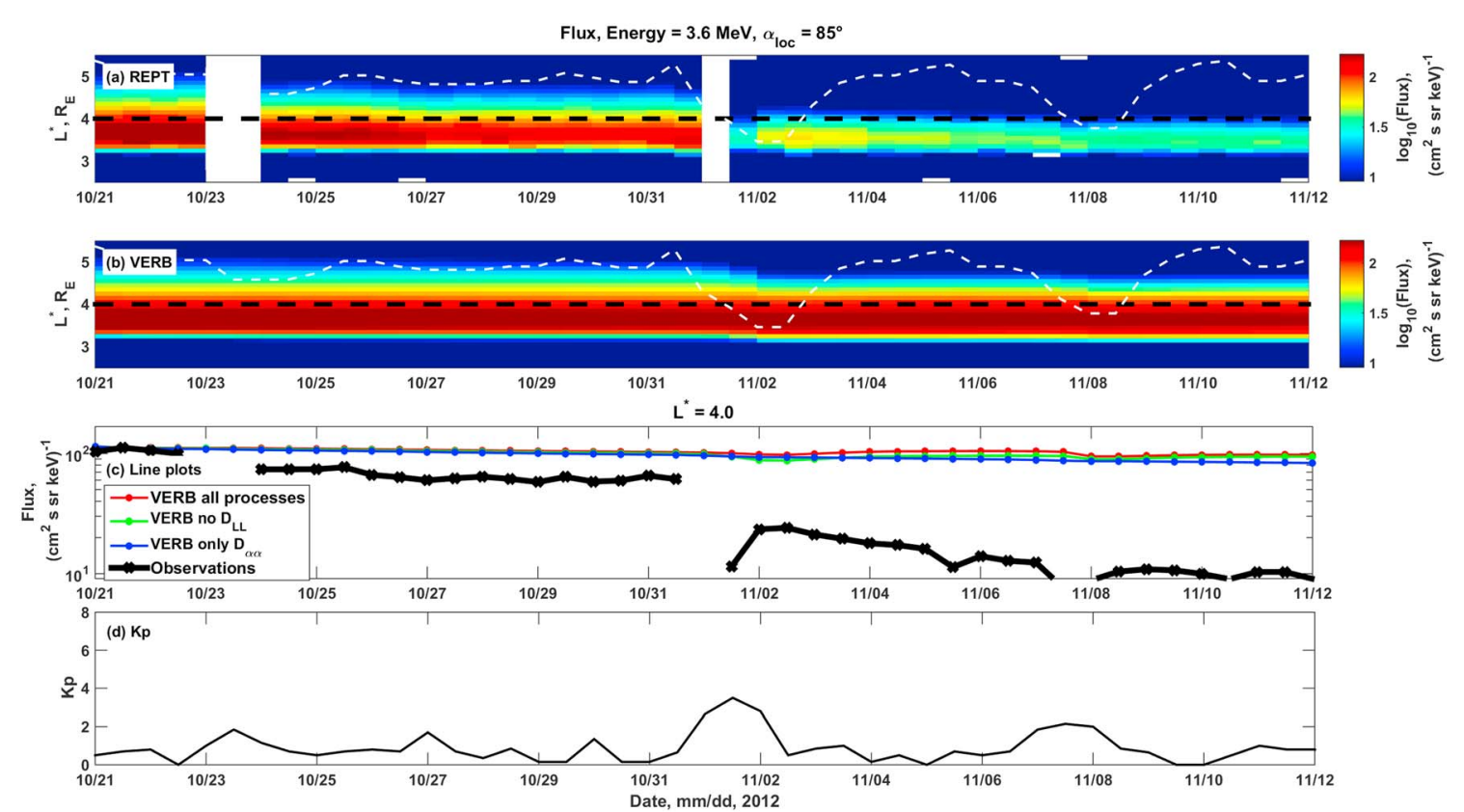

Figure 4. Ultrarelativistic electron flux at $3.6 \mathrm{MeV}, \alpha_{\mathrm{loc}}=85^{\circ}$ for 30 days. (a) REPT flux observations, (b) corresponding VERB code simulation based on boundary conditions from REPT data, (c) line plots of the flux at fixed $L^{*}=4$, and (d) $K p$ index; the red line represents the simulation with radial, energy, and pitch angle diffusion; the green line represents the same simulation excluding radial diffusion; the blue line denotes the simulation based on pitch angle diffusion only, and the black line denotes the observation data.

To analyze the contribution of pitch angle scattering alone, we performed the simulation based on equation (4):

$$
\frac{\partial f}{\partial t}=\left.\frac{1}{T(\alpha) \sin (2 \alpha)} \frac{\partial}{\partial \alpha}\right|_{p, \mathrm{~L}^{*}} T(\alpha) \sin (2 \alpha)\left(\left.D_{\alpha \alpha} \frac{\partial f}{\partial \alpha}\right|_{p, \mathrm{~L}^{*}}\right)-\frac{f}{\tau_{l c}}
$$

The calculations were made for a period of 30 days during the moderate geomagnetic conditions after the 13 October 2012 storm, from 21 October 2012 to 21 November 2012. The initial conditions were the same for each simulation period of 30 days and were based on REPT observations.

Figure 4 shows the 30 day period of the $3.6 \mathrm{MeV}$ flux observation (Figure 4a) in comparison with the simulation based on equation (2) (Figure 4b), including all diffusion processes. The line plots in Figure $4 \mathrm{c}$ illustrate the simulations based on equations (2)-(4) and observation of the electron flux at fixed $\mathrm{L}^{*}=4.0$. The different colored lines (red, green, and blue) represent fluxes based on the above described simulations. It can be clearly seen at the end of the period considered that all three simulations overestimate the measurements from the REPT instrument (black line in Figure 4c). It means that none of the processes can explain the rapid decreasing of the ultrarelativistic electron observations.

As expected, the simulation based on equation (4) generally shows a lower level of fluxes as it excludes any processes that can transport electrons into the heart of the radiation belts or accelerate electrons from the lower energies. However, even simulations with only pitch angle diffusion are insufficient to explain the observed loss of electrons. This contrasts with the favorable comparison between the observed and modeled PSDs on 19 September 2012 using the initial conditions from 7 September 2012 by Thorne et al. [2013a], which is most likely a result of competing source and loss processes that may be operating at that time or may be simply a result of the short time period considered in that study.

The differences between the simulations are no larger than a factor of $\sim 2$ at the end of the simulation. In contrast, the observed flux is significantly less. This suggests that modeled loss rates are not high enough to reproduce the observed values. To investigate the possible causes for the disagreement in ultrarelativistic energies, we conducted a detailed analysis of the modeled and observed flux variations for different electron energies. 


\section{AGU Journal of Geophysical Research: Space Physics}
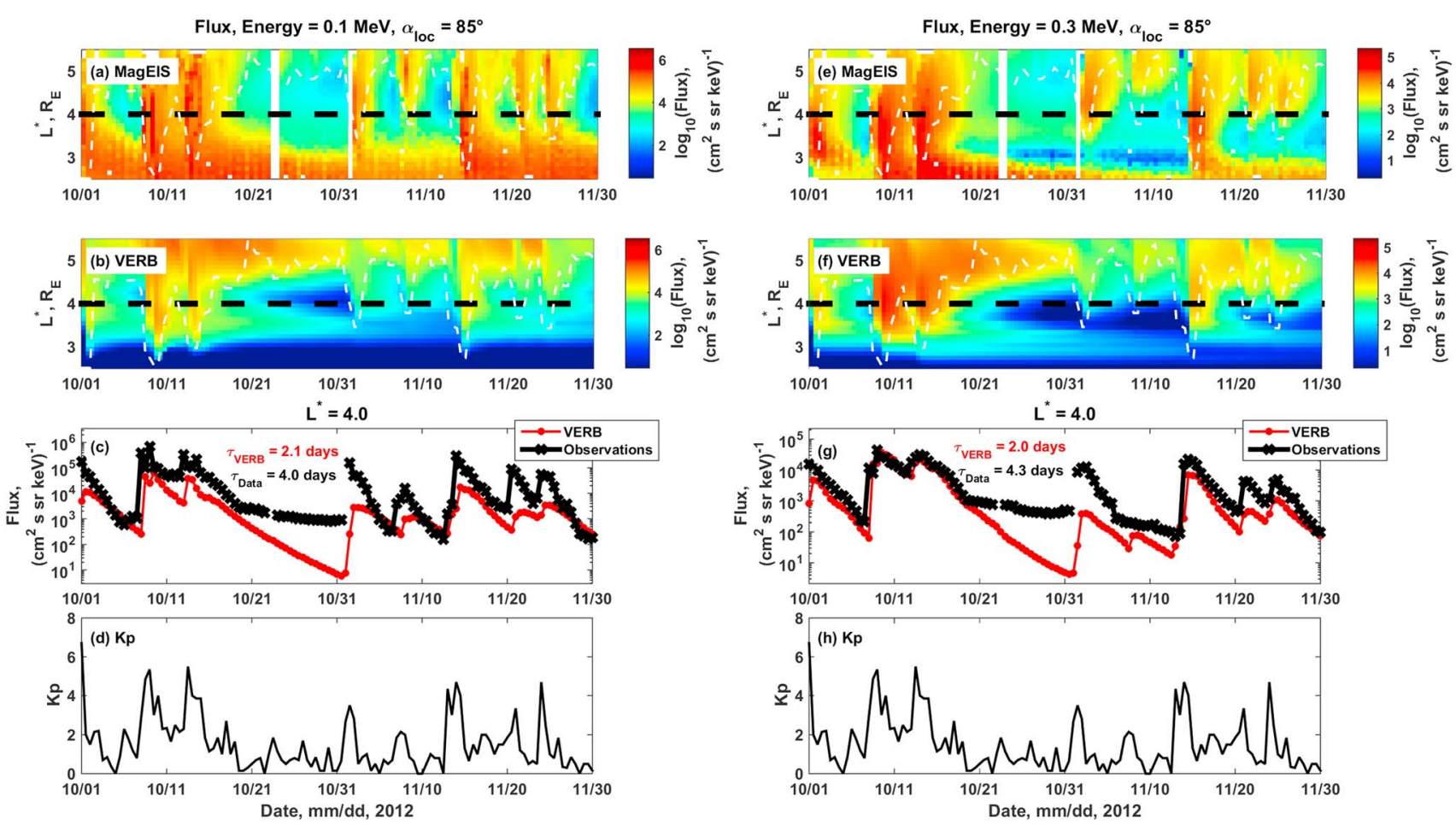

Figure 5. Energetic electron flux for 60 days. MagElS flux observations (a) $0.1 \mathrm{MeV}$ and (e) $0.3 \mathrm{MeV}, \alpha_{\mathrm{loc}}=85^{\circ}$. (b and f) Corresponding VERB code simulation based on boundary conditions from MagEIS data. The simulation is the same as that presented in Figure 2. (c and g) The line plots of the flux at fixed $\mathrm{L}^{*}=4.0$ are shown: red line represents the simulation and black line represents the observation data. ( $\mathrm{d}$ and $\mathrm{h}$ ) The $K p$ index variation is also shown.

Figure 5 shows the energetic electron fluxes for the 60 day period starting from 1 October 2012 of the 1 year simulation that was discussed above in section 4.2. The comparison of the $0.1 \mathrm{MeV}$ fluxes between the MagEIS observation and the VERB code simulation based on equation (2) is shown in the left column (Figures $5 \mathrm{a}$ and $5 \mathrm{~b}$, respectively) and for the energy $0.3 \mathrm{MeV}$ in the right column (Figures $5 \mathrm{e}$ and $5 \mathrm{f}$ ). To exclude the possible chorus acceleration and focus on the hiss wave contribution, we calculated the empirical decay rates at fixed $\mathrm{L}^{*}=4.0$ inside the plasmasphere for the period from 17 to 30 October 2012 . The difference between VERB code-simulated $\left(\tau_{\text {VERB }}\right)$ and data-observed $\left(\tau_{\text {Data }}\right)$ decay rates is moderate and no larger than by factor of $\sim 2$. For the energy $0.1 \mathrm{MeV}$, the ratio $\tau_{\mathrm{VERB}} / \tau_{\text {Data }} \simeq 0.5\left(\tau_{\mathrm{VERB}}=2.1\right.$ days, $\tau_{\text {Data }}=4.0$ days). For energy $0.3 \mathrm{MeV}, \tau_{\text {VERB }} / \tau_{\text {Data }} \simeq 0.5$ ( $\tau_{\text {VERB }}=2.0$ days, $\tau_{\text {Data }}=4.3$ days $)$.

Comparison of the fluxes along the wide range of $L^{*}$ (Figures $5 a, 5 b, 5 e$, and $5 f$ ) shows a small discrepancy between simulations and measurements. The overestimated measurements around $\mathrm{L}^{*}=3-4$ for the period from 15 to 31 October are likely due to contamination by X-rays produced by the multi-MeV electrons (S. Claudepierre, personal communication 2014). The observation of the multi-MeV electrons in Figure 7e shows the remnant belt located in the same area as the slight contamination in Figure $5 \mathrm{a}$ and $5 \mathrm{e}$, for this same period. Discrepancies between the model and observations are also likely to be due to the neglect of convection in the code that provides a significant acceleration mechanism at these energies. The difference at the lower $L$ shells below $L^{*}=3$ may be also due to the inapplicability of Brautigam and Albert's [2000] diffusion coefficients at these energies and $L$ shells. In addition, the discrepancies at the outer boundary may be due to the simplified method that uses only a variation of fluxes at $0.9 \mathrm{MeV}$ following Brautigam and Albert [2000]. However, the modeled steady state decay rates appear to be rather similar to the observed decay rates (Figures $5 \mathrm{c}$ and $5 \mathrm{~g}$ ).

Figure 6 is similar to Figure 5, but it presents the relativistic electron fluxes for energies $0.5 \mathrm{MeV}$ (Figures 6a-6d) and $0.9 \mathrm{MeV}$ (Figures $6 \mathrm{e}-6 \mathrm{~h}$ ). The simulation flux value reached the observed enhancement during the 12 October 2013 storm and followed the observed dynamics of the relativistic electron belt variation. Accurate simulations of the observed acceleration events in November likely require empirical data of the 


\section{AGU Journal of Geophysical Research: Space Physics}
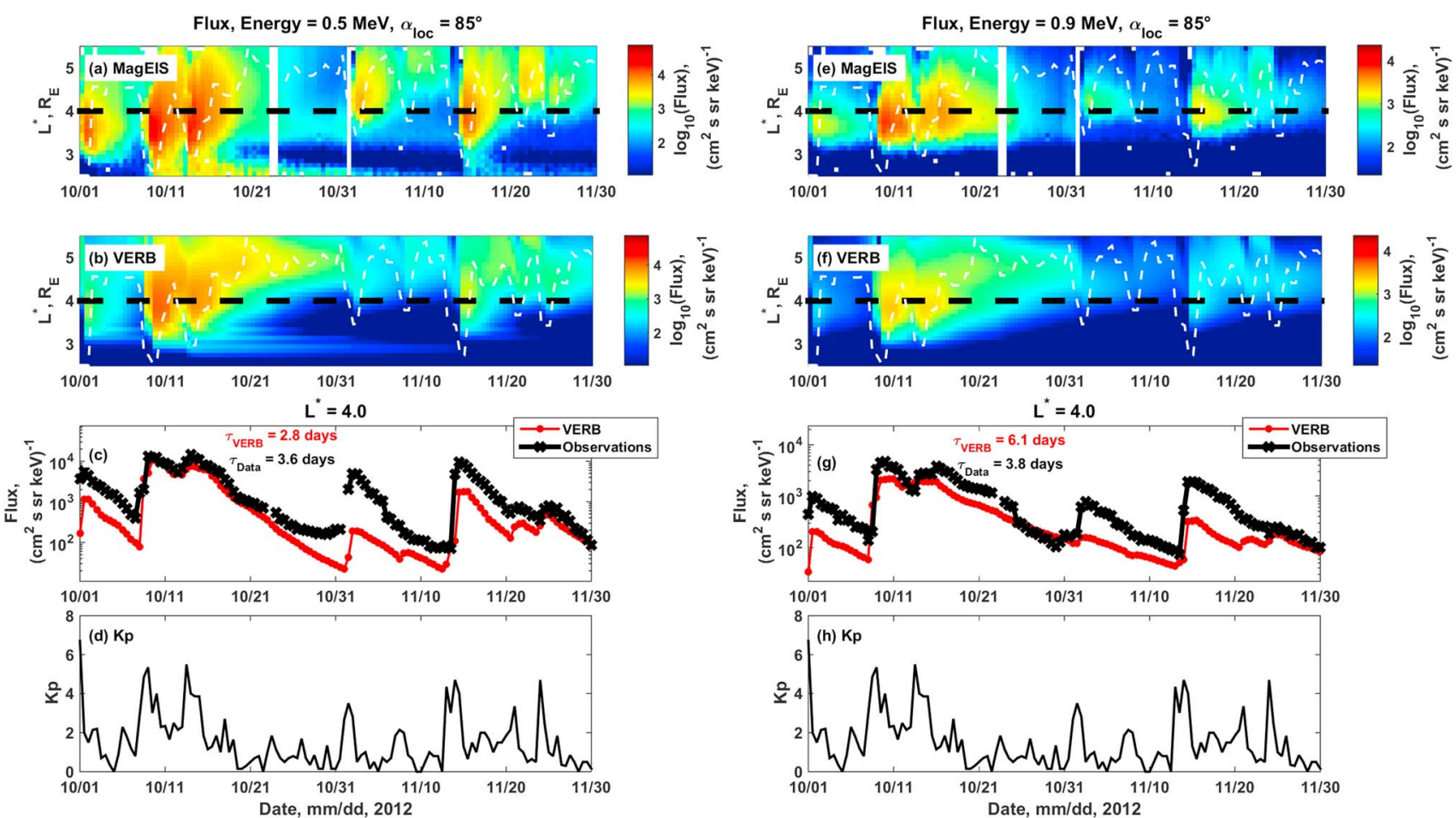

Figure 6. Same figure as in Figure 5 but for relativistic electron flux for (a-d) $0.5 \mathrm{MeV}$ and (e-h) $0.9 \mathrm{MeV}$ energies, $\alpha_{\mathrm{loc}}=85^{\circ}$.
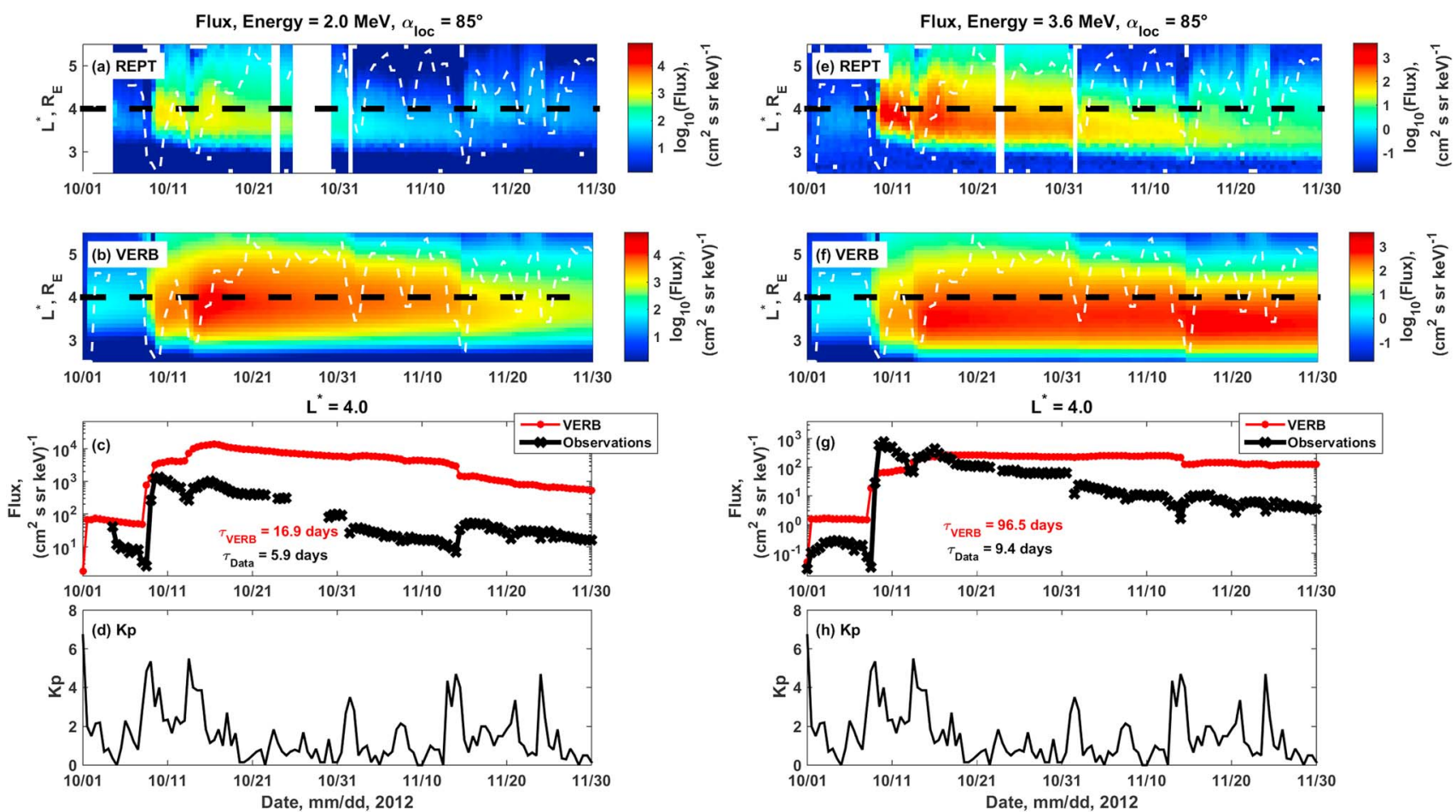

Figure 7. Same figure as in Figure 5 but for energies (a-d) $2.0 \mathrm{MeV}$ and (e-h) $3.6 \mathrm{MeV}, \alpha_{\mathrm{loc}}=85^{\circ}$. The simulation is the same as presented in Figure 3 and is based on boundary conditions from REPT data. Observations are from the REPT instrument. 
plasmapause location and plasma density. However, the decay rates are estimated for the period from 17 to 30 October and are reproduced rather well. For the energy $0.5 \mathrm{MeV}$, the ratio is $\tau_{\mathrm{VERB}} / \tau_{\text {Data }} \simeq 0.8\left(\tau_{\mathrm{VERB}}=2.8\right.$ days, $\tau_{\text {Data }}=3.6$ days $)$. For the energy $0.9 \mathrm{MeV}, \tau_{\mathrm{VERB}} / \tau_{\text {Data }} \simeq 1.6$ ( $\tau_{\mathrm{VERB}}=6.1$ days, $\tau_{\text {Data }}=3.8$ days $)$. The difference between simulated and observed decay rates is smaller than a factor of $\sim 2$.

Figure 7 shows the comparison for the electron fluxes at energies $2.0 \mathrm{MeV}$ (Figures $7 \mathrm{a}-7 \mathrm{~d}$ ) and $3.6 \mathrm{MeV}$ (Figures 7e-7h). The observed and simulated line plots in Figures $7 \mathrm{c}$ and $7 \mathrm{~g}$ show a significant difference in electron lifetimes. For $2.0 \mathrm{MeV}$, the electron model underestimates the decay rates, and the ratio is $\tau_{\mathrm{VERB}} / \tau_{\text {Data }} \simeq 2.9\left(\tau_{\mathrm{VERB}}=16.9\right.$ days, $\tau_{\text {Data }}=5.9$ days $)$. The discrepancy between the model and observations is even higher at energy of $3.6 \mathrm{MeV}$. The ratio is $\tau_{\mathrm{VERB}} / \tau_{\text {Data }} \simeq 10.3$ ( $\tau_{\mathrm{VERB}}=96.5$ days, $\tau_{\text {Data }}=9.4$ days $)$. These results clearly show that at ultrarelativistic energies, the model that includes only hiss scattering is not capable of reproducing observed decay rates.

The current wave model was applied for all energies in the long-period simulations. The simulations provide good agreement with energetic and relativistic electron dynamics. However, the significant discrepancy for the ultrarelativistic electrons indicates that other loss processes may be acting on this population.

\section{Summary and Discussion}

In this study, we used the VERB code to model the dynamics of the outer electron radiation belt during a long period of time, which included various levels of geomagnetic activity. We tested our model, which included an improved parameterization of the plasmaspheric hiss, as follows: first, we compared the model with the relativistic electron flux variation observed by CRRES. The updated model showed an agreement with previous studies. To obtain a comprehensive picture of the radiation belt dynamics, we performed simulations using the VERB code based on 1 year of Van Allen Probes measurements. This comparison also showed a relatively good qualitative agreement between the simulated and observed relativistic electron fluxes and PSD ( $\sim 1 \mathrm{MeV}, \mu=700 \mathrm{MeV} / \mathrm{G})$. A comparison of the decay rates produced in the simulations and the observations showed relatively good agreement for an even broader range of energies, including energetic $(>0.1 \mathrm{MeV})$ and relativistic $(\sim 1 \mathrm{MeV}, \mu=700 \mathrm{MeV} / \mathrm{G})$ electrons. However, the simulated PSD for higher $\mu=3500 \mathrm{MeV} / \mathrm{G}$ and fluxes for the ultrarelativistic energies were significantly different to those observed. Detailed analysis of the decay rates for different energies showed that the observed ultrarelativistic energy fluxes were lost on shorter time scales than predicted by the model. This can be due to missing physics or inaccurate wave parameterizations.

We first discuss the wave and plasma parameters and how they can affect the simulation results. Wave amplitude can significantly influence the diffusion coefficients, since scattering rates are proportional to wave amplitude squared. Several studies [Cattell et al., 2008; Cully et al., 2008] found that the chorus wave amplitude can reach values up to $\sim 240 \mathrm{mV} / \mathrm{m}$. In this case, nonlinear effects can potentially play some role and should be taken into account in the future. The wave normal angle distribution is also a substantial factor that could affect the diffusion coefficients. It was shown that highly oblique chorus waves could increase the pitch angle scattering rates near the loss cone by as much as 1 order of magnitude, i.e., decrease the electron lifetimes [Artemyev et al., 2012]. Nevertheless, it is likely that this effect will influence a wide range of energies, and since we see the discrepancy between the observed and modeled decay rates at only multi-MeV energies, it is not likely that the observed difference can be explained by this effect. Our simulations also show that most of the decay occurs inside of the plasmasphere where hiss waves are present. For hiss waves, it was shown that changing the wave normal angle distribution does not substantially change the diffusion coefficients [Artemyev et al., 2013]. We should also note that our assumed wave normal distributions for hiss are consistent with observations and ray tracing [Agapitov and Artemyev, 2013; Thorne et al., 2013a]. It was recently shown that plasmaspheric hiss frequencies could be lower than typical values of $100-2000 \mathrm{~Hz}$ [ $L i$ et al., 2013]. In this case, the decay rates for ultrarelativistic electrons can be lower, but the difference in decay rates will be less than a factor of 2 . A detailed investigation of this effect will be a subject for future research. Finally, plasma density is another factor that influences the scattering rates. We used statistical plasma models to compute the scattering rates for the VERB simulations. However, during specific events, the plasma density could significantly differ from these models. Thorne et al. [2013b] found that during the 9 October 2012 storm, there was a long enhanced period of convective activity. Enhanced convection led to the substantial decrease of plasma 
density, which caused the increase in local acceleration of ultrarelativistic electrons. Thus, the changes in plasma density can only affect particular events but are not likely to significantly change the long-term balance between sources and losses.

Summarizing the discussion above, changing whistler wave and plasma parameters is not likely to produce the observed loss of multi-MeV electrons, and simulations exploring the sensitivity to different models of waves will be a subject of future study.

Thorne and Kennel [1971], Summers and Thorne [2003], Li et al. [2007], and Ukhorskiy et al. [2010] suggested that EMIC waves may provide efficient scattering mechanisms for relativistic electrons. Most recently, Shprits et al. [2013] suggested that EMIC waves may provide effective pitch angle scattering for ultrarelativistic electrons, while such scattering may not be very efficient for the relativistic electrons. In particular, Shprits et al. [2013] showed that the unusual dynamics of the ultrarelativistic electrons [Baker et al., 2013a] can be reproduced when EMIC wave scattering is taken into account. The suggestion that EMIC waves affect ultrarelativistic electrons is confirmed by a recent study [Usanova et al., 2014], in which the observed electron pitch angle distributions were consistent with scattering by EMIC waves. To produce loss for the nearly equatorially particles, the presence of additional gyroscattering by hiss, chorus [Li et al., 2007], other wave modes, or bounce scattering by waves [Shprits et al., 2009b] may be required. In this study, we did not include the effect of EMIC waves since continuous wave observations at all MLT and L shells are not available, and interactions with EMIC waves strongly depend on the assumed wave spectrum. The development and testing of the parameterizations of EMIC waves will be a subject of future research.

\section{Acknowledgments}

The authors used geomagnetic indices provided by OMNIWeb (http://omniweb. gsfc.nasa.gov/form/dx1.html) and are grateful to the RBSP-ECT team for the provision of Van Allen Probes observations (http://www.rbsp-ect.lanl. gov/). We acknowledge S.G. Claudepierre and J.B. Blake for their helpful discussion. The diffusion coefficients used in the VERB code are available on the Space Environment Modeling Group website (ftp://rbm.epss.ucla.edu/). In addition, authors are appreciative of the valuable comments of the reviewers, whose contribution deserves special acknowledgment. The research of K. Orlova was supported by the NASA Living with a Star Jack Eddy Postdoctoral Fellowship Program, administered by the University Corporation for Atmospheric Research. This research was supported by the NSF grant AGS-1203747, NASA grant NNX13AE34G, and received funding support from the UC Office of the President, UC Lab Fees Research Program grant 12-LR-235337.

Michael Balikhin thanks Allison Jaynes and another reviewer for their assistance in evaluating this paper.

\section{References}

Agapitov, O., and A. Artemyev (2013), Statistics of whistler mode waves in the outer radiation belt: Cluster STAFF-SA measurements, J. Geophys. Res. Space Physics, 118, 3407-3420, doi:10.1002/jgra.50312.

Albert, J. M., N. P. Meredith, and R. B. Horne (2009), Three-dimensional diffusion simulation of outer radiation belt electrons during the 9 October 1990 magnetic storm, J. Geophys. Res., 114, A09214, doi:10.1029/2009JA014336.

Artemyev, A., O. Agapitov, H. Breuillard, V. Krasnoselskikh, and G. Rolland (2012), Electron pitch-angle diffusion in radiation belts: The effects of whistler wave oblique propagation, Geophys. Res. Lett., 39, L08105, doi:10.1029/2012GL051393.

Artemyev, A. V., O. V. Agapitov, and D. Mourenas (2013), Storm-induced energization of radiation belt electrons: Effect of wave obliquity, Geophys. Res. Lett., 40, 4138-4143, doi:10.1002/grl.50837.

Baker, D. N., et al. (2013a), A long-lived relativistic electron storage ring embedded in Earth's outer Van Allen belt, Science, 340(6129), 186-190, doi:10.1126/science.1233518.

Baker, D. N., et al. (2013b), The Relativistic Electron-Proton Telescope (REPT) instrument on board the Radiation Belt Storm Probes (RBSP) spacecraft: Characterization of Earth's radiation belt high-energy particle populations, Space Sci. Rev., 179, 337-381, doi:10.1007/s11214-012-9950-9.

Blake, J. B., et al. (2013), The Magnetic Electron Ion Spectrometer (MagEIS) instruments aboard the Radiation Belt Storm Probes (RBSP) spacecraft, Space Sci. Rev., 179, 383-421, doi:10.1007/s11214-013-9991-8.

Boyd, A. J., H. E. Spence, S. G. Claudepierre, J. F. Fennell, J. B. Blake, D. N. Baker, G. D. Reeves, D. L. Turner, and H. O. Funsten (2014), Quantifying the radiation belt seed population in the 17 March 2013 electron acceleration event, Geophys. Res. Lett., 41, 2275-2281, doi:10.1002/ 2014GL059626.

Brautigam, D. H., and J. M. Albert (2000), Radial diffusion analysis of outer radiation belt electrons during the October 9, 1990, magnetic storm, J. Geophys. Res., 105(A1), 291-309, doi:10.1029/1999JA900344.

Carpenter, D. L., and R. R. Anderson (1992), An ISEEE/whistler model of equatorial electron density in the magnetosphere, J. Geophys. Res., 97(A2), 1097-1108, doi:10.1029/91JA01548.

Cattell, C., et al. (2008), Discovery of very large amplitude whistler-mode waves in Earth's radiation belts, Geophys. Res. Lett., 35, L01105, doi:10.1029/2007GL032009.

Cully, C. M., J. W. Bonnell, and R. E. Ergun (2008), THEMIS observations of long-lived regions of large-amplitude whistler waves in the inner magnetosphere, Geophys. Res. Lett., 35, L17S16, doi:10.1029/2008GL033643.

Denton, R. E., J. D. Menietti, J. Goldstein, S. L. Young, and R. R. Anderson (2004), Electron density in the magnetosphere, J. Geophys. Res., 109, A09215, doi:10.1029/2003JA010245.

Denton, R. E., K. Takahashi, I. A. Galkin, P. A. Nsumei, X. Huang, B. W. Reinisch, R. R. Anderson, M. K. Sleeper, and W. J. Hughes (2006), Distribution of density along magnetospheric field lines, J. Geophys. Res., 111, A04213, doi:10.1029/2005JA011414.

Glauert, S. A., R. B. Horne, and N. P. Meredith (2014), Three-dimensional electron radiation belt simulations using the BAS Radiation Belt Model with new diffusion models for chorus, plasmaspheric hiss, and lightning-generated whistlers, J. Geophys. Res. Space Physics, 119, 268-289, doi:10.1002/2013JA019281.

Green, J. C., and M. G. Kivelson (2001), A tale of two theories: How the adiabatic response and ULF waves affect relativistic electrons, J. Geophys. Res., 106(A11), 25,777-25,791, doi:10.1029/2001JA000054.

Horne, R. B., N. P. Meredith, R. M. Thorne, D. Heynderickx, R. H. A. lles, and R. R. Anderson (2003), Evolution of energetic electron pitch angle distributions during storm time electron acceleration to megaelectronvolt energies, J. Geophys. Res., 108(A1), 11-13, doi:10.1029/ 2001JA009165

Kennel, C. F., and F. Engelmann (1966), Velocity space diffusion from weak plasma turbulence in a magnetic field, Phys. Fluids, 9(2377), 2377-2388, doi:10.1063/1.1761629.

Kim, K.-C., and Y. Shprits (2013), Long-term relativistic radiation belt electron responses to GEM magnetic storms, J. Atmos. Sol. Terr. Phys., 100-101, 59-67, doi:10.1016/j.jastp.2013.04.007. 
Kim, K.-C., D.-Y. Lee, Y. Shprits, H.-J. Kim, and E. Lee (2011a), Electron flux changes in the outer radiation belt by radial diffusion during the storm recovery phase in comparison with the fully adiabatic evolution, J. Geophys. Res., 116, A09229, doi:10.1029/2011 JA016642.

Kim, K.-C., Y. Shprits, D. Subbotin, and B. Ni (2011b), Understanding the dynamic evolution of the relativistic electron slot region including radial and pitch angle diffusion, J. Geophys. Res., 116, A10214, doi:10.1029/2011JA016684.

Kim, K.-C., Y. Shprits, D. Subbotin, and B. Ni (2012), Relativistic radiation belt electron responses to GEM magnetic storms: Comparison of CRRES observations with 3-D VERB simulations, J. Geophys. Res., 117, A08221, doi:10.1029/2011JA017460.

Li, W., Y. Y. Shprits, and R. M. Thorne (2007), Dynamic evolution of energetic outer zone electrons due to wave-particle interactions during storms, J. Geophys. Res., 112, A10220, doi:10.1029/2007JA012368.

$\mathrm{Li}, \mathrm{W}$., et al. (2013), An unusual enhancement of low-frequency plasmaspheric hiss in the outer plasmasphere associated with substorm-injected electrons, Geophys. Res. Lett., 40, 3798-3803, doi:10.1002/grl.50787.

Mauk, B. H., N. J. Fox, S. G. Kanekal, R. L. Kessel, D. G. Sibeck, and A. Ukhorskiy (2013), Science objectives and rationale for the Radiation Belt Storm Probes mission, Space Sci. Rev., 179(1-4), 3-27, doi:10.1007/s11214-012-9908-y.

Millan, R. M., and D. N. Baker (2012), Acceleration of particles to high energies in Earth's radiation belts, Space Sci. Rev., 173(1-4), 103-131, doi:10.1007/s11214-012-9941-x.

O'Brien, T. P. (2014), Breaking all the invariants: Anomalous electron radiation belt diffusion by pitch angle scattering in the presence of split magnetic drift shells, Geophys. Res. Lett., 41, 216-222, doi:10.1002/2013GL058712.

O'Brien, T. P., and M. B. Moldwin (2003), Empirical plasmapause models from magnetic indices, Geophys. Res. Lett., 30(4), 1152, doi:10.1029/ 2002 GL016007.

Orlova, K., M. Spasojevic, and Y. Shprits (2014), Activity-dependent global model of electron loss inside the plasmasphere, Geophys. Res. Lett., 41, 3744-3751, doi:10.1002/2014GL060100.

Reeves, G. D., K. L. McAdams, R. H. W. Friedel, and T. P. O'Brien (2003), Acceleration and loss of relativistic electrons during geomagnetic storms, Geophys. Res. Lett., 30(10), 1529, doi:10.1029/2002GL016513.

Reeves, G. D., Y. Chen, G. S. Cunningham, R. W. H. Friedel, M. G. Henderson, V. K. Jordanova, J. Koller, S. K. Morley, M. F. Thomsen, and S. Zaharia (2012), Dynamic Radiation Environment Assimilation Model: DREAM, Space Weather, 10, S03006, doi:10.1029/2011SW000729.

Reeves, G. D., et al. (2013), Electron acceleration in the heart of the Van Allen radiation belts, Science, 341(6149), 991-994, doi:10.1126/ science.1237743.

Roederer, J. G. (1970), Dynamics of Geomagnetically Trapped Radiation, Phys. and Chem. in Space, vol. 2, p. 166, Springer, Berlin, doi:10.1007/ 978-3-642-49300-3.

Rothwell, P., and C. E. Mcllwain (1960), Magnetic storms and the Van Allen radiation belts-Observations from satellite $1958 \varepsilon$ (Explorer IV), J. Geophys. Res., 65(3), 799-806, doi:10.1029/JZ065i003p00799.

Russell, C. T., and R. M. Thorne (1970), On the structure of the inner magnetosphere, in Cosmic Electrodynamics, vol. 1, pp. 67-89, D. Reidel, Dordrecht-Holland.

Schulz, M., and L. J. Lanzerotti (1974), Particle diffusion in the radiation belts, in Physics and Chemistry in Space, vol. 7, 218 pp., Springer, Berlin, doi:10.1007/978-3-642-65675-0.

Shprits, Y. Y., R. M. Thorne, G. D. Reeves, and R. Friedel (2005), Radial diffusion modeling with empirical lifetimes: Comparison with CRRES observations, Ann. Geophys., 23(4), 1467-1471, doi:10/5194/angeo-23-1467-2005.

Shprits, Y. Y., R. M. Thorne, R. B. Horne, S. A. Glauert, M. Cartwright, C. T. Russell, D. N. Baker, and S. G. Kanekal (2006a), Acceleration mechanism responsible for the formation of the new radiation belt during the 2003 Halloween solar storm, Geophys. Res. Lett., 33, L05104, doi:10.1029/2005GL024256.

Shprits, Y. Y., W. Li, and R. M. Thorne (2006b), Controlling effect of the pitch angle scattering rates near the edge of the loss cone on electron lifetimes, J. Geophys. Res., 111, A12206, doi:10.1029/2006JA011758.

Shprits, Y. Y., S. R. Elkington, N. P. Meredith, and D. A. Subbotin (2008a), Review of modeling of losses and sources of relativistic electrons in the outer radiation belt I: Radial transport, J. Atmos. Sol. Terr. Phys., 70(14), 1679-1693, doi:10.1016/j.jastp.2008.06.008.

Shprits, Y. Y., D. A. Subbotin, N. P. Meredith, and S. R. Elkington (2008b), Review of modeling of losses and sources of relativistic electrons in the outer radiation belt II: Local acceleration and loss, J. Atmos. Sol. Terr. Phys., 70(14), 1694-1713, doi:10.1016/j.jastp.2008.06.014.

Shprits, Y. Y., D. Subbotin, and B. Ni (2009a), Evolution of electron fluxes in the outer radiation belt computed with the VERB code, J. Geophys. Res., 114, A11209, doi:10.1029/2008JA013784.

Shprits, Y. Y., L. Chen, and R. M. Thorne (2009b), Simulations of pitch angle scattering of relativistic electrons with MLT-dependent diffusion coefficients, J. Geophys. Res., 114, A03219, doi:10.1029/2008JA013695.

Shprits, Y. Y., D. Subbotin, A. Drozdov, M. E. Usanova, A. Kellerman, K. Orlova, D. N. Baker, D. L. Turner, and K.-C. Kim (2013), Unusual stable trapping of the ultrarelativistic electrons in the Van Allen radiation belts, Nat. Phys., 9(11), 699-703, doi:10.1038/nphys2760.

Spence, H. E., et al. (2013), Science goals and overview of the Energetic particle, Composition, and Thermal plasma (ECT) suite on NASA's Radiation Belt Storm Probes (RBSP) mission, Space Sci. Rev., 179, 311-336, doi:10.1007/s11214-013-0007-5.

Stratton, J. M., R. J. Harvey, and G. A. Heyler (2014), Mission overview for the Radiation Belt Storm Probes mission, in The Van Allen Probes Mission, edited by N. Fox and J. L. Burch, pp. 29-57, Springer US, Boston, Mass., doi:10.1007/s11214-012-9933-x.

Su, Z., F. Xiao, H. Zheng, and S. Wang (2011), CRRES observation and STEERB simulation of the 9 October 1990 electron radiation belt dropout event, Geophys. Res. Lett., 38, L06106, doi:10.1029/2011GL046873.

Subbotin, D. A., and Y. Y. Shprits (2009), Three-dimensional modeling of the radiation belts using the Versatile Electron Radiation Belt (VERB) code, Space Weather, 7, S10001, doi:10.1029/2008SW000452.

Subbotin, D. A., Y. Y. Shprits, and B. Ni (2011a), Long-term radiation belt simulation with the VERB 3-D code: Comparison with CRRES observations, J. Geophys. Res., 116, A12210, doi:10.1029/2011JA017019.

Subbotin, D. A., Y. Y. Shprits, M. Gkioulidou, L. R. Lyons, B. Ni, V. G. Merkin, F. R. Toffoletto, R. M. Thorne, R. Horne, and M. K. Hudson (2011b), Simulation of the acceleration of relativistic electrons in the inner magnetosphere using RCM-VERB coupled codes, J. Geophys. Res., 116, A08211, doi:10.1029/2010JA016350.

Summers, D., and R. M. Thorne (2003), Relativistic electron pitch-angle scattering by electromagnetic ion cyclotron waves during geomagnetic storms, J. Geophys. Res., 108(A4), 1143, doi:10.1029/2002JA009489.

Thorne, R. M., and C. F. Kennel (1971), Relativistic electron precipitation during magnetic storm main phase, J. Geophys. Res., 76(19), 4446-4453, doi:10.1029/JA076i019p04446.

Thorne, R. M., et al. (2013a), Evolution and slow decay of an unusual narrow ring of relativistic electrons near L 3.2 following the September 2012 magnetic storm, Geophys. Res. Lett., 40, 3507-3511, doi:10.1002/grl.50627.

Thorne, R. M., et al. (2013b), Rapid local acceleration of relativistic radiation-belt electrons by magnetospheric chorus, Nature, 504, 411-414, doi:10.1038/nature12889. 
Tsyganenko, N. A. (1989), A magnetospheric magnetic field model with a warped tail current sheet, Planet. Space Sci., 37(1), 5-20, doi:10.1016/0032-0633(89)90066-4.

Tu, W., X. Li, Y. Chen, G. D. Reeves, and M. Temerin (2009), Storm-dependent radiation belt electron dynamics, J. Geophys. Res., 114 , A02217, doi:10.1029/2008JA013480.

Tu, W., G. S. Cunningham, Y. Chen, M. G. Henderson, E. Camporeale, and G. D. Reeves (2013), Modeling radiation belt electron dynamics during GEM challenge intervals with the DREAM3D diffusion model, J. Geophys. Res. Space Physics, 118, 6197-6211, doi:10.1002/ jgra.50560.

Tu, W., G. S. Cunningham, Y. Chen, S. K. Morley, G. D. Reeves, J. B. Blake, D. N. Baker, and H. Spence (2014), Event-specific chorus wave and electron seed population models in DREAM3D using the Van Allen Probes, Geophys. Res. Lett., 41, 1359-1366, doi:10.1002/2013GL058819.

Turner, D. L., et al. (2014), Competing source and loss mechanisms due to wave-particle interactions in Earth's outer radiation belt during the 30 September to 3 October 2012 geomagnetic storm, J. Geophys. Res. Space Physics, 119, 1960-1979, doi:10.1002/2014JA019770.

Ukhorskiy, A. Y., Y. Y. Shprits, and B. J. Anderson (2010), Rapid scattering of radiation belt electrons by storm-time EMIC waves, Geophys. Res. Lett., 37, L09101, doi:10.1029/2010GL042906.

Usanova, M. E., et al. (2014), Effect of EMIC waves on relativistic and ultrarelativistic electron populations: Ground-based and Van Allen Probes observations, Geophys. Res. Lett., 41, 1375-1381, doi:10.1002/2013GL059024.

Vampola, A. L., J. V. Osborn, and B. M. Johnson (1992), CRRES magnetic electron spectrometer AFGL-701-5A (MEA), J. Spacecr. Rockets, 29(4), 592-595, doi:10.2514/3.25504

Van Allen, J. A., and L. A. Frank (1959), Radiation around the Earth to a radial distance of 107,400 km, Nature, 183, 430-434, doi:10.1038/ $183430 \mathrm{a} 0$.

Vernov, S. N., E. V. Gorchakov, S. N. Kuznetsov, Y. I. Logachev, E. N. Sosnovets, and V. G. Stolpovsky (1969), Particle fluxes in the outer geomagnetic field, Rev. Geophys., 7(1,2), 257-280, doi:10.1029/RG007i001p00257. 